\title{
Evaluation of Morphological Changes in Breast Cancer: Influence of Fractal Meta-Analysis
}

\author{
Seyed Abbas Mirmalek ${ }^{1 凶}$, Niloofar sadat Yarandi ${ }^{2}$, Mohammad Tafazoli Shadpour ${ }^{3}$, Minoo Saatian ${ }^{4}$, Homa Raeesi ${ }^{5}$, \\ Esmaieel Jafargholi Rangraz ${ }^{3}$, Masoud Salehi ${ }^{6}$, Seyed Alireza Salimi Tabatabaee ${ }^{7}$

\footnotetext{
${ }^{1}$ General Surgery Department, Islamic Azad University of Medical Sciences and Health Services, Boali Hospital, Tehran, Iran. ${ }^{2}$ Islamic Azad University of Medical Sciences and Health Services, Boali Hospital, Tehran, Iran.

${ }^{3}$ Biomedical Engineering, Amirkabir University, Tehran, Iran.

${ }^{4}$ Pathology Department, Islamic Azad University of Medical Sciences and Health Services, Boali Hospital, Tehran, Iran ${ }^{5}$ Pathology ward, Kasra Hospital, Tehran, Iran.

${ }^{6}$ Department of Biostatistics, Faculty of Management and Medical Informatics, Tehran University of Medical Sciences, Tehran, Iran. ${ }^{7}$ Medical Student, Islamic Azad University, Tehran Medical Branch, Tehran, Iran.
}

\section{Dear Editor}

$\mathrm{B}$ reast cancer is the most common cancer among women in developed countries, and the National Cancer Institute has predicted that more than two million women would be diagnosed with breast cancer from 1990 to 2010 in the United states $[1,2]$. Some researchers believe that the incidence of breast cancer is increasing in many countries, although in some cases the mortality rate may be fixed or slightly reduced [3]. Due to its increasing incidence all over the world and being a medical concern globally, finding predictive methods for its diagnosis as well as its prognosis are of great importance [4].

Studying breast cancer, many researchers currently use a novel method known as fractal geometry $[5,6]$. Fractals were complex geometrical structures. Developed in 1975, science of fractal images not only is applied in engineering but also has gained importance in medical sciences [7]. Fractals were used for diagnosis of bone diseases, modeling of bone structures, and analysis of bone changes, as well as analysis of heart rhythm and rate and diagnosis of heart diseases. Nevertheless, fractal geometry can be a good diagnostic and

\section{GMJ}

@2013 Galen Medical Journal

Fax: +98 7312227091

PO Box 7461686688

Email:info@gmj.ir prognostic tool wherever structural disorders and malfunctions exist [7]. Since cancer is considered as a type of cellular and histological anarchy, the dimensions of these irregularities can be shown through their quantification and reporting in the form of fractal numbers [8]. This study has investigated the relationship between the fractal numbers in patients with breast cancer and the histopathological grade.

This cross-sectional study was performed on 60 women that were selected through convenient sampling from 1000 women with ductal carcinoma of the breast that referred to BooAli hospital in Tehran, Iran, from June 2010 to October 2013. All patients underwent local anesthesia and the biopsy was taken from both breasts and underwent histopathological analyses. After being kept in buffered formaldehyde $(\mathrm{pH}=7.2)$ and creating paraffin blocks, three $5 \mu \mathrm{m}$ sections were prepared from each sample and the slides were stained with hematoxylin-eosin (H\&E) and examined by light microscope. In addition to their pathology slides, photographs were taken to assess the fractal number by special software. To this end, the Box-Counting method was used in MATLAB-R2007a software.

\footnotetext{
Correspondence to:

Seyed Abbas Mirmalek, General Surgery Department, Islamic Azad University of Medical Sciences and Health Services, Boali Hospital, Tehran, Iran Telephone Number: (+98) 2188787561 Email Address :sam@mirmalek.net
} 
Analysis was done by using independent t-test, ANOVA, Fisher's exact test, and chisquare in SPSS 15.0. The significant difference was set at $\mathrm{P}<0.05$.

The mean age of the studied women was $52.48 \pm 10.83$ years. The grade of the carcinoma was 2 in 38 women (63.3\%) and 3 in 22 women $(36.7 \%)$. The mean size of the tumor was $2.8 \pm 1.48 \mathrm{~cm}$ and the mean fractal number was $1.95 \pm 0.03$ in all cases. A significant difference existed between the mean fractal numbers of grade $2(1.94 \pm 0.03)$ and grade 3 $(1.98 \pm 0.004)(\mathrm{P}=0.001)$.

Fractal is a multi-component geometrical image which can be divided into patches each patch being similar to the "whole" image [7]. It is hard to believe that fractal as a highly complicated and difficult concept which is applied at the highest levels of mathematics can be easily used in a study [8]. In general, despite its complexity, fractal can easily be used in medical sciences [9]. Previous stud- ies have shown that the fractal number can differentiate benign or malignant tumors $[8$, 9]; but the present study sought to use fractal number to realize the grade of malignancy and obtained interesting results; the higher the fractal number, the increased tumor grade. Geometrically, fractal is an object with three features, self-similarity, high complexity in micro scale, and with dimension not an integer $[7,8]$. Like many studies, we did not find an association between the risk factors and the fractal number $[10,11]$.

The findings of this study indicated a significant relationship between the fractal number and the histopathological grade of the tumor, for example the fractal number was significantly increased by increasing the histopathological grade of the breast cancer. Thus, a fractal number could be a quantitative concept of tumor grade and can be measured as a predictor of breast cancer prognosis.

References

1. Jemal A, Siegel R, Ward E, Hao Y, Xu J, Thun MJ. Cancer statistics, 2009. CA Cancer J Clin. 2009;59(4):225-49.

2. Qaseem A, Snow V, Sherif K, Aronson $\mathrm{M}$, Weiss KB, Owens DK. Screening mammography for women 40 to 49 years of age: a clinical practice guideline from the American College of Physicians. Ann Intern Med. 2007;146(7): 511-5.

3. Smigal C, Jemal A, Ward E, Cokkinides V, Smith R, Howe HL, et al. Trends in breast cancer by race and ethnicity: update 2006 . CA Cancer J Clin. 2006; 56(3):168-83.

4. Humphrey LL, Helfand M, Chan BK, Woolf SH. Breast cancer screening: a summary of the evidence for the U.S. Preventive Services Task Force. Ann Intern Med. 2002;137(5 Part 1):347-60.

5. Vahabi M. Breast cancer screening methods: a review of the evidence. Health Care Women Int. 2003;24(9):773-93.

6. Gail MH, Costantino JP, Pee D, Bondy M, Newman L, Selvan M, et al. Projecting individualized absolute invasive breast cancer risk in African American women. J Natl Cancer Inst. 2007; 99(23):1782-92.

7. Nguyen T, Rangayyan R. Shape Analysis of Breast Masses in Mammograms via the Fractal Dimension. Conf Proc IEEE Eng Med Biol Soc. 2005;3:3210-3.

8. Dey P, Mohanty SK. Fractal dimensions of breast lesions on cytology smears. Diagn Cytopathol. 2003;29(2):85-6.

9. Li H, Giger ML, Olopade OI, Lan L. Fractal analysis of mammographic parenchymal patterns in breast cancer risk assessment. Acad Radiol. 2007;14(5):513-21.

10. Crişan DA, Lesaru M, Dobrescu R, Vasilescu C. Fractal analysis in the diagnosis of breast tumors. Chirurgia (Bucur). 2007;102(1):2730.

11. Krasowska M, Grzywna ZJ, Mycielska ME, Djamgoz MB. Fractal analysis and ionic dependence of endocytotic membrane activity of human breast cancer cells. Eur Biophys J. 2009;38(8):1115-25. 